\title{
Quasar polarization properties
}

from Robert Carswell

Two surveys of quasars recently undertaken by astronomers at Steward Observatory in Arizona and reported in the Astrophysical Journal (279, 465 and 485; 1984) give us a comprehensive overview of the polarization properties of the quasar continuum radiation at optical wavelengths. Some of the results are valuable confirmations of old beliefs, such as the strong link between high polarization and rapid variability at optical and radio frequencies, but the work is notable for the new material it provides. One of the most important conclusions to emerge is that quasars can be divided into two classes, those with linear polarization of less than 2 per cent and a small fraction (about 1 per cent of the total number) with polarization greater than 3 per cent and up to an observed maximum of 30 per cent. In the case of the weakly polarized sources, the polarization appears to be aligned with the extended radio structure, where it is present, and there is evidence that the polarization is greater at short wavelengths than at long ones.

One of the popular mechanisms for producing continuum radiation in quasars and Seyfert galaxies (which behave very much like mini-quasars) is radiation from relativistic electrons which are constrained by a magnetic field (synchrotron radiation). If the magnetic field is ordered, the radiation emitted by this process should be highly polarized - up to about 70 per cent - and the degree of polarization should not depend strongly on wavelength. Astronomers seeking to confirm the importance of synchrotron radiation at optical wavelengths were rewarded with a small number of quasars which show strong rapidly-variable polarization, but it soon became clear that in most cases the polarization was below the few per cent detection limit of the early studies. Moreover, while synchrotron radiation at optical wavelengths adequately explains those quasars which show high polarization, for the large majority it is not clear that this mechanism is operating, or even that the optical continuum is necessarily non-thermal in origin.

The Steward Observatory polarization studies go some way towards answering some of the outstanding questions. First, they show that linear polarization is a feature of the continuum radiation at optical wavelengths of all quasars, albeit at a rather lower level than might have been expected. Second, on the basis of the new results, several proposed explanations for the polarization properties of quasars can now be discounted. It has been suggested that all quasars have the same basic structure, but that the properties observed are determined by our orientation with respect to some anisotropic component. However, we would not then expect to see a clear differentiation in polarization properties between the bulk of quasars and the few highly polarized ones. Nor does this observation support a model in which a polarized (synchrotron?) component interacts with some other (thermal?) continuum, with the relative strengths varying from quasar to quasar. Rather, the two classes of polarization appear to reflect differences in the intrinsic properties of the quasars.

The observations on the lowpolarization quasars are, in fact, in reasonable agreement with the predictions of only one model - polarization by dust. (A similar model is used to describe our own Galaxy.) Light scattered by dust grains becomes polarized, independent of the alignment of the grains, but dependent on the angle through which the light is scattered. If it were not possible to resolve the dust-scattering region from the continuum source, as would be the case for quasars, the object itself would appear polarized, provided the dust did not have a spherically symmetric distribution. Thus, if the system is rotating and the dust is in a disc or flattened sphere, for example, we should see a net polarization except in the relatively few cases in which the axis of rotation is aligned towards us. Moreover, since dust grains scatter light more effectively at short wavelengths than at long wavelengths, shortwavelength light is more polarized. This is exactly what Stockman and his colleagues at Steward Observatory have seen. They also find some alignment of the radio structure with the polarization, assuming that the rotation axis of the dusty disc can be aligned with the radio structure.

This is a very important result, and serves to strengthen the link between quasars and Seyfert galaxies, whose emission properties have been explained in terms of dust obscuration. As far as understanding the nature of the continuum radiation, however, it is perhaps the least helpful of the explanations which have been considered. Since the dust is almost certainly outside the continuum source and will modify any intrinsic polarization properties the continuum might have, the polarization observations provide few constraints on a model of the continuum radiation. Even synchrotron radiation from regions with a very disordered magnetic field remains a possibility. Future studies of the highly-polarized and rapidly-variable quasars will no doubt yield further valuable information for that class, but it is not clear how they relate to the low-polarization objects.

Robert Carswell is at the Institute of Astronomy, The Observatories, Madingley Road, Cambridge CB3 OHA.

\section{Drug delivery}

\section{Old drugs in new clothing}

\section{from Gregory Gregoriadis}

WITH the cost of the development of new drugs forever mounting, it may be more sensible to make better use of those already available. To this end, the controlled delivery of current drugs by biodegradable carriers could decrease their side effects, dosage and frequency of administration. A recent meeting* was devoted to the consideration of alternative carrier systems for drugs, including man-made materials, such as polymers and liposomes, and natural materials, notably antibodies. They can be used in the form of implants, releasing drugs at rates conducive to optimal action, often through controlled degradation, or be employed in systems of targeted delivery.

There has been no shortage of ingenuity in controlling degradation of implants. $\mathbf{J}$. Heller (SRI, Menlo Park), for instance, uses poly(ortho)ester polymers with acidsensitive linkages in their backbone, incorporating acidic salts in a highly hydrophobic environment. Since only salt on the sur-

* 'Macromolecules as drugs and as carriers for biologically active materials', New York, 26-28 March, was organized by Drs York Academy of Sciences. face of the polymer is exposed to water, polymer erosion by hydrolysis should occur only at the surface. Therefore, drug release can be controlled over periods ranging from hours to months by the amount of salt present. Controlled release can also be achieved by a combination of drug diffusion and polymer biodegradation. Thus, microspheres made of copolymers of lactic acid and glycolic acid will release a drug as a function both of its concentration and of certain physical parameters of the polymer; injected microspheres loaded with a contraceptive steroid led to predictable and efficacious levels of the steroid in volunteers for up to six months (D.H. Lewis, Stolle Research and Development Corporation, Alabama). Even non-biodegradable materials, such as polymethyl methacrylate or polystyrene, can be converted into useable carriers by introducing biodegradable sections along the polymer chain (W.J. Bailey, University of Maryland).

An alternative to the passive entrapment of drugs in a polymer matrix is to arrange for them to be bound on to the matrix and 